\title{
Revisiting RFID Link Budgets for Technology Scaling: Range Maximization of RFID Tags
}

\author{
Ritochit Chakraborty, Sumit Roy and Vikram Jandhyala \\ Department of Electrical Engineering, University of Washington, Seattle \\ \{ritochit, sroy, vj\}@u.washington.edu
}

\begin{abstract}
Passive RFID tags are traditionally assumed to be downlink limited since typical tag sensitivity is considerably poorer than reader sensitivity, due to stringent power limitations. On the other hand, semi-passive tags are generally uplink limited since tag and reader sensitivity are comparable. In this paper, it is demonstrated that judicious choice and use of IC impedance for backscatter modulation will be needed to simultaneously maximize tag read and write ranges as passive tag designs improve. Optimal backscatter modulation indices for amplitude-shift-keying are derived for range maximization of next generation low-power RFID tags.
\end{abstract}

\section{INTRODUCTION}

Passive RFID tags are (strongly) power limited and depend on rectification of downlink signal from the reader to operate their circuitry. It has long been held that availability of power for tag IC operation is the system limiter, as opposed to the detector sensitivity for decoding of queries; i.e., passive tag systems are downlink (range) limited [1]. On the other hand, semi-passive tags that are battery-assisted [1] incorporate a power source (e.g. a coin cell) in the tag IC, but still use backscattered communication on the uplink. As a result, sensitivity of semi-passive tags (whose operation is not limited by power considerations) approach that of the reader detector; thus, RFID systems based on semi-passive tags are uplink (and not downlink) limited, with the backscattered power at the reader constituting the limit.

Given the extreme constraints on available power for passive tags, whether they are able to respond at all to reader query depends on the ability of the reader to transfer sufficient power on the downlink to support particular circuit functions such as the backscatter modulation on the uplink. This induces a maximum distance for reliable reader-to-tag communication that is denoted as the 'write' (downlink) range. For passive tags, the write range is limited not by the sensitivity to decoding the tag query signal, but by the power requirement for tag ICs. On the uplink, the 'read' (uplink) range is determined by the reader detectability of the tag data and the received backscattered power. Clearly, the smaller of these two ranges determines system performance and passive RFID systems have historically been downlink limited [1]. The above highlights an important facet of RFID systems that appears to have been under-appreciated in the existing literature - the fundamental asymmetry of the uplink and downlink ranges

This work was supported in part by NSF under grant number ECCS0824265 . at which information may be reliably communicated. Thus, a system design objective is to improve the write range for passive tags to match the read range. With continuing advancements in IC technology, RFID tags that consume much less power than their predecessors [2-4] are being designed, that directly contributes to this. For example, [5] proposed a novel RFID tag that consumes only $2.7 \mu \mathrm{W}$, significantly lower than the $25 \mu \mathrm{W}$ in [6] or the $16.7 \mu \mathrm{W}$ in [3].

This paper examines the problem of optimizing the range for amplitude-shift-keying (ASK) modulation on the uplink. ASK requires two impedance states for the tag IC to achieve backscatter modulation [2-4]. Each modulation is characterized by an index that, in turn, determines the power backscattered to the reader. As tag IC power thresholds decrease, a link budget analysis shows that a cross-over between uplink and downlink range emerges. In other words, future passive tags with improved sensitivity may be read range limited.

A recent work [7] proposes optimal ON/OFF resistance for the ASK modulated passive RFID system. This scheme maximizes the harvestable power for the tag IC in order to enhance identification range between the tag and the reader. Range estimation has not been undertaken in [7] to highlight the actual improvement in range brought about by the proposed optimal configuration. It is shown that the effective backscattered power deteriorates under antenna mismatch conditions. However, as outlined in this paper, a trade-off emerges between uplink and downlink ranges because of technology scaling in the absence of mismatch as well. Thus, future passive RFID tags may become read range limited even when tag antenna is matched. The aforementioned trade-off is exploited in range maximization of RFID tags.

The organization of the paper is as follows. Sections II and III outline link budget analysis based downlink and uplink range estimation for ASK modulation. Section IV then discusses concurrent maximization of both read and write ranges and Section $\mathrm{V}$ offers observations and concluding remarks.

\section{Downlink RANGe Estimation}

Figure 1 depicts the equivalent circuit model for the RFID tag. The tag IC is modeled as a complex impedance $Z_{I C}$ consisting of a resistance and a capacitance such that

$$
Z_{I C}=R_{I C}-\frac{j}{\omega C_{I C}}
$$

The Thevenin equivalent model for the antenna consists of a 


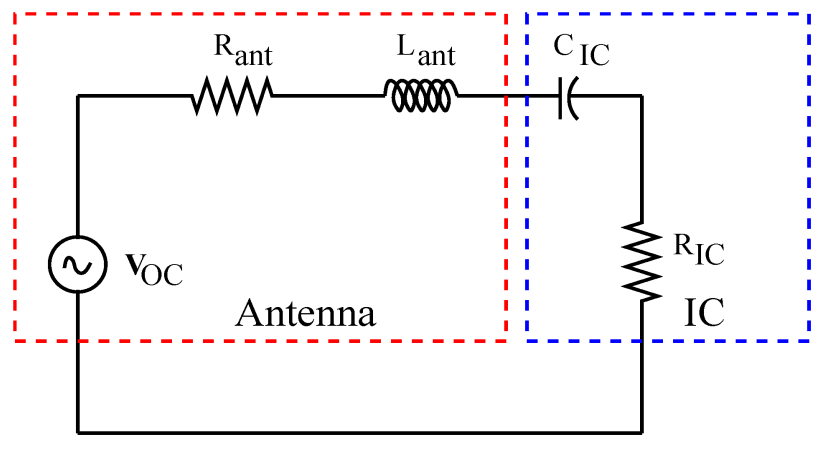

Fig. 1. Equivalent circuit model for RFID tag showing tag antenna and IC.

voltage source $V_{O C}$ in series with a complex impedance $Z_{a n t}$ where

$$
Z_{\text {ant }}=R_{a n t}+j \omega L_{a n t}
$$

and $R_{\text {ant }}$ combines the radiation and ohmic loss resistances. When possible, to ensure maximum power transfer to the tag, the antenna is designed for $Z_{\text {ant }}=Z_{I C}^{*}$. Otherwise, a powermatching network is placed between the antenna and tag to accomplish conjugate match [4]. Thus, $R_{a n t}=R_{I C}$ in the absence of any modulation.

Figure 2 provides a simple model for backscatter modulation by insertion of a modulating resistance in either series or parallel with the IC. The IC capacitance and antenna inductance cancel each other at the frequency of operation and are not shown in Fig. 2. Series or parallel modulation alters the tag IC resistance to $R_{I C, e q}=m R_{a n t}$, where $m$ is the 'impedance modulation index'. Placement of a series modulating resistance ensures $m>1$ while parallel placement ensures $0<m<1$. Thus, the series and parallel modulating resistances are respectively

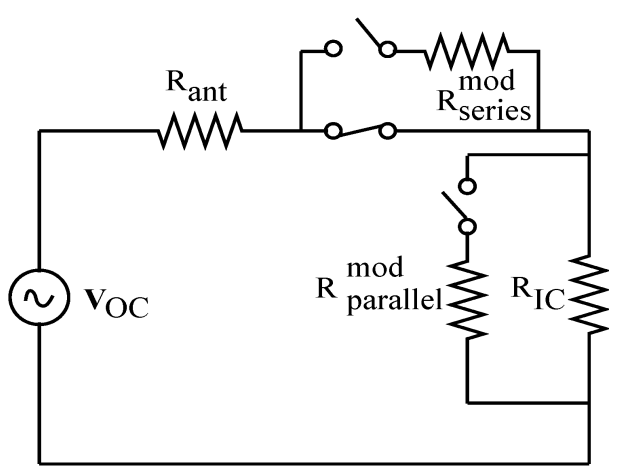

Fig. 2. A switching mechanism depicting series and parallel resistive modulation of $R_{I C}$. Only one switch is closed at a time.

$$
\begin{array}{r}
R_{\text {series }}^{\text {mod }}=(m-1) R_{\text {ant }} \\
R_{\text {parallel }}^{\text {mod }}=\left(\frac{m}{1-m}\right) R_{\text {ant }}
\end{array}
$$

The peak current $I_{p k}$ flowing through the tag IC can be computed as

$$
I_{p k}=\frac{V_{O C}}{Z_{a n t}+Z_{I C}}=\frac{V_{O C}}{(1+m) R_{a n t}}
$$

and the corresponding total power supplied is

$$
P_{\text {total }, I C}=\frac{1}{2}\left|I_{p k}\right|^{2} R_{I C, e q}=\frac{\left|V_{O C}\right|^{2}}{2 R_{a n t}} \frac{m}{(1+m)^{2}}
$$

However, part of this power is dissipated in the modulation resistor and only the remaining is available to supply the IC. For parallel modulation $(0<m<1)$, the actual usable power delivered to the IC is

$$
P_{\text {usable }, I C}^{\text {parallel }}=m P_{\text {total }, I C}=\frac{\left|V_{O C}\right|^{2}}{2 R_{\text {ant }}} M_{\text {parallel }}
$$

where

$$
M_{\text {parallel }}=\left(\frac{m}{1+m}\right)^{2}
$$

For series modulation $(m>1)$, the actual usable power delivered to the IC is

$$
P_{\text {usable }, I C}^{\text {series }}=\frac{P_{\text {total }, I C}}{m}=\frac{\left|V_{O C}\right|^{2}}{2 R_{\text {ant }}} M_{\text {series }}
$$

where

$$
M_{\text {series }}=\left(\frac{1}{1+m}\right)^{2}
$$

In equations (7) and (9), $M_{\text {parallel }}$ and $M_{\text {series }}$ are respective power scaling factor (PSF) as a function of impedance modulation index $m$. Thus, the combined PSF for usable power supply to tag IC $M_{I C}$ is

$$
M_{I C}= \begin{cases}\left(\frac{m}{1+m}\right)^{2}, & \text { for } 0 \leq m \leq 1 \\ \left(\frac{1}{1+m}\right)^{2}, & \text { for } m>1\end{cases}
$$

For a more general analysis, it is assumed that the tag IC resides in two impedance states state 1 and state 2 for backscatter modulation where the impedances are, respectively,

$$
\begin{aligned}
& Z_{I C}^{\text {state } 1}=m_{1} R_{\text {ant }}-j \omega L_{\text {ant }} \\
& Z_{I C}^{\text {state } 2}=m_{2} R_{\text {ant }}-j \omega L_{\text {ant }}
\end{aligned}
$$

The tag IC impedances in equation (11) may be the result of (a) parallel modulation in both states, (b) series modulation in both states, (c) parallel modulation in one state and series in the other. While only parallel or series modulation are intuitive, it may also be possible to operate with a mismatch in both states by alternating between parallel and series modulation when the tag antenna has been designed for $Z_{\text {ant }}=Z_{I C}^{*}$.

Assuming that the tag encodes backscattered data as FM0 baseband, the IC resides in each of its two impedance states an equal amount of time [3], and the time-average power delivered to the tag IC for rectification is 
$P_{\text {tag }}^{\text {parallel }}=0.5 * \frac{\left|V_{O C}\right|^{2}}{2 R_{\text {ant }}}\left[\left(\frac{m_{1}}{1+m_{1}}\right)^{2}+\left(\frac{m_{2}}{1+m_{2}}\right)^{2}\right]$

for parallel modulation in both states, while it is

$P_{\text {tag }}^{\text {series }}=0.5 * \frac{\left|V_{O C}\right|^{2}}{2 R_{\text {ant }}}\left[\left(\frac{1}{1+m_{1}}\right)^{2}+\left(\frac{1}{1+m_{2}}\right)^{2}\right]$

for series modulation in both states, and finally, it is

$$
P_{\text {tag }}^{\text {mixed }}=0.5 * \frac{\left|V_{O C}\right|^{2}}{2 R_{a n t}}\left[\left(\frac{m_{1}}{1+m_{1}}\right)^{2}+\left(\frac{1}{1+m_{2}}\right)^{2}\right]
$$

for parallel modulation in statel and series modulation in state 2 .

In general, the RFID reader can be assumed to reside in the far-field of the tag. In compliance with FCC regulations for unlicensed transmitters, the reader is assumed to emit 1 W of power with a transmit antenna gain $G_{t x}$ of $6 \mathrm{dBi}$ [1]. This translates to an effective isotropic radiated power (EIRP) $P_{\text {eirp }}$ of $4 \mathrm{~W}$. The reader antenna considered in this work is circularly polarized with $0 \mathrm{~dB}$ axial ratio.

For reader-tag downlink distance $r_{\text {write }}$, the impinging power density $P_{d e n}$ at the tag is given by

$$
P_{\text {den }}=\frac{P_{\text {eirp }}}{4 \pi r_{\text {write }}^{2}}
$$

Thus, the peak value of the incident electric field $E_{i n c}$ along the tag axis is

$$
E_{\text {inc }}=\sqrt{Z_{0} \eta P_{\text {den }}}=\frac{1}{2 r_{\text {write }}}\left(\frac{Z_{0} \eta P_{\text {eirp }}}{\pi}\right)^{1 / 2}
$$

where $Z_{0}$ is free-space impedance and the factor $\eta$ accounts for the polarization mismatch loss due to the linearly polarized tag antenna.

The induced open-circuit port voltage $V_{O C}$ at the tag antenna is proportional to $E_{i n c}$ and is denoted as

$$
V_{O C}=\alpha_{t a g} E_{i n c}=\frac{\alpha_{t a g}}{2 r_{w r i t e}}\left(\frac{Z_{0} \eta P_{\text {eirp }}}{\pi}\right)^{1 / 2}
$$

where the vector effective length $\alpha_{t a g}$ is dependent on of the geometrical layout of the tag antenna $[8,9]$. Thus, $\alpha_{t a g}$ is a function of $\theta$ and $\phi$ only.

Thus, if the tag sensitivity is $P_{t a g}^{0}$, then based on the selected modulation scheme, the write (downlink) range may be

$$
\begin{aligned}
D_{\text {write }}^{\text {parallel }}= & {\left[\frac{\left|\alpha_{\text {tag }}\right|}{4}\right]\left(\frac{Z_{0} \eta P_{\text {eirp }}}{\pi R_{\text {ant }} P_{\text {tag }}^{0}}\right)^{1 / 2} } \\
& \sqrt{\left[\left(\frac{m_{1}}{1+m_{1}}\right)^{2}+\left(\frac{m_{2}}{1+m_{2}}\right)^{2}\right]}
\end{aligned}
$$

or,

$$
\begin{array}{r}
D_{\text {write }}^{\text {series }}=\left[\frac{\left|\alpha_{\text {tag }}\right|}{4}\right]\left(\frac{Z_{0} \eta P_{\text {eirp }}}{\pi R_{a n t} P_{\text {tag }}^{0}}\right)^{1 / 2} \\
\sqrt{\left[\left(\frac{1}{1+m_{1}}\right)^{2}+\left(\frac{1}{1+m_{2}}\right)^{2}\right]}
\end{array}
$$

or,

$$
\begin{array}{r}
D_{\text {write }}^{\text {mixed }}=\left[\frac{\left|\alpha_{\text {tag }}\right|}{4}\right]\left(\frac{Z_{0} \eta P_{\text {eirp }}}{\pi R_{\text {ant }} P_{\text {tag }}^{0}}\right)^{1 / 2} \\
\sqrt{\left[\left(\frac{m_{1}}{1+m_{1}}\right)^{2}+\left(\frac{1}{1+m_{2}}\right)^{2}\right]}
\end{array}
$$

\section{UPLINK RANGE ESTIMATION}

The power reflection coefficients $\rho_{1}$ and $\rho_{2}$ for modulated tag impedances [3] are given, respectively, as

$$
\begin{aligned}
& \rho_{1}=\frac{Z_{I C}^{\text {state } 1}-Z_{\text {ant }}^{*}}{Z_{I C}^{\text {state } 1}+Z_{\text {ant }}}=\frac{m_{1}-1}{m_{1}+1} \\
& \rho_{2}=\frac{Z_{I C}^{\text {state } 2}-Z_{\text {ant }}^{*}}{Z_{I C}^{\text {state } 2}+Z_{\text {ant }}}=\frac{m_{2}-1}{m_{2}+1}
\end{aligned}
$$

for the two states of the tag IC impedance.

If $I_{\text {state } 1}$ and $I_{\text {state } 2}$ denote the currents induced at the tag antenna terminals in state 1 and state 2 respectively, then for tag-reader uplink distance $r_{\text {read }}$, the modulated backscattered electric fields at the reader are given, respectively, as

$$
\begin{aligned}
& E_{b s}^{\text {state1 }}=I_{\text {state } 1} \frac{E_{a}}{I_{a}}=I_{\text {match }}\left(1-\rho_{1}\right) \frac{E_{a}}{I_{a}} \\
& E_{b s}^{\text {state2 }}=I_{\text {state } 2} \frac{E_{a}}{I_{a}}=I_{\text {match }}\left(1-\rho_{2}\right) \frac{E_{a}}{I_{a}}
\end{aligned}
$$

where $I_{\text {match }}$ denotes the current induced at the tag antenna terminals for a conjugate match between the tag antenna and its load [10], and is given by

$$
I_{\text {match }}=\frac{V_{O C}}{2 R_{\text {ant }}}
$$

Also, for the set of equations in equation (22), $E_{a}$ denotes the field radiated by the tag antenna when the current at its terminals is $I_{a}$ [8] and no external excitation is applied to it. For free-space propagation, the ratio $\left|E_{a} / I_{a}\right|$ is given by

$$
\left|\frac{E_{a}}{I_{a}}\right|=\frac{Z_{0}\left|\alpha_{\text {tag }}\right|}{2 \lambda r_{\text {read }}}
$$

Assuming (a) no polarization mismatch at the reader antenna, and (b) conjugate match between the reader antenna and its load, the induced open-circuit voltages $V_{1}$ and $V_{2}$ at the reader antenna are given, respectively, as

$$
\begin{aligned}
& V_{1}=\alpha_{r d} E_{b s}^{\text {state1 }}=\alpha_{r d} I_{\text {match }}\left(1-\rho_{1}\right) \frac{E_{a}}{I_{a}} \\
& V_{2}=\alpha_{r d} E_{b s}^{\text {state2 }}=\alpha_{r d} I_{\text {match }}\left(1-\rho_{2}\right) \frac{E_{a}}{I_{a}}
\end{aligned}
$$


Since the vector effective lengths $\alpha_{t a g}$ and $\alpha_{r d}$ are proportional to the square root of their respective antenna gains in a specific direction [8], their relationship can be expressed as

$$
\frac{\alpha_{r d}}{\alpha_{t a g}}=\sqrt{\frac{G_{r x}}{G_{t a g}}}
$$

with $G_{r x}$ representing the reader receive antenna gain in a specific direction, and $G_{t a g}$ denoting the tag antenna gain in the same direction.

\section{A. Uplink Range}

The uplink performance is determined by the reader's ability to decode the tag data which depends on the received backscattered signal power at the reader. In turn, the latter determines the achievable bit error rate (BER) for the specific modulation on the uplink [11].

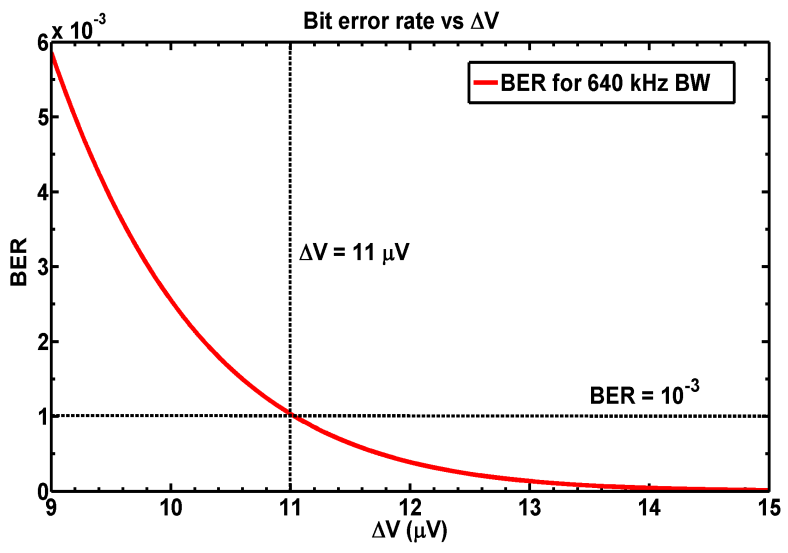

Fig. 3. Bit error rate vs. $\Delta V$ for $640 \mathrm{kHz}$ tag bandwidth.

Since the typical RFID reader is monostatic (one RF chain for both transmit and receive as shown in Fig. 4) - it continues to transmit an unmodulated carrier on the downlink while simultaneously listening to the modulated tag response on the uplink. There is always some leakage from transmit to receiver chain consisting of both the (a) downlink $\mathrm{CW}$ signal component as well as (b) the transmit LO phase noise [1]. For a reader transmitting $30 \mathrm{dBm} \mathrm{CW}$ on downlink, the signal leakage is typically $15 \mathrm{~dB}$ below the transmitted signal, i.e., around $15 \mathrm{dBm}$. Thus for any decoding of the tag backscatter modulated signal, this $\mathrm{CW}$ component must be removed, which is achieved by dc blocking in the reader. *

The primary performance limiter on the uplink is the LO phase noise leaking from the transmit chain which overshadows the thermal noise component. Per [1], the phase noise power spectral density is typically around $-115 \mathrm{dBc} / \mathrm{Hz}$ relative to the $\mathrm{CW}$ signal power at $640 \mathrm{kHz}$ offset. Thus, for 640 (40) $\mathrm{kHz}$ tag signal bandwidth, the total LO noise power is $(-115+59)=-56(-68) \mathrm{dBc}$ relative to the $\mathrm{CW}$ signal. Hence, for a $\mathrm{CW}$ signal component of $15 \mathrm{dBm}$, the phase noise power is approximately $(15-56)=-41 \mathrm{dBm}(-53 \mathrm{dBm})$.

* Any CW component at the center frequency appears as a dc shift after demodulation in the reader.
For BER determination, this phase noise needs to be converted into a voltage in the baseband receiver. The antenna reflection is not in phase with the local oscillator signal as it has to travel down cables to the antenna and back as shown in Fig. 4. The total delay for the transmit signal to reach the antenna, get reflected and finally reach the mixer, depicted in Fig. 4 as $\tau$ ns, introduces variation in the absolute phase of the reflected signal. This phase variation, in turn, affects the output voltage of the mixer that is fed by the local oscillator. In this paper, in accordance with the analysis in [1], it is estimated that the phase noise is reduced by a factor of $50 \mathrm{~dB}$ in being converted to amplitude noise. Thus, the equivalent amplitude noise at the receiver is $(-41-50)=-91 \mathrm{dBm}(-103 \mathrm{dBm})$. If the leakage power is $P_{l e a k}=-91 \mathrm{dBm}$, then the BER is

$$
B E R=\frac{1}{2} \operatorname{erfc}\left(\frac{\left|V_{1}-V_{2}\right| / 2}{2 \sqrt{2} \sqrt{P_{\text {leak }}}}\right)
$$

with erfc(.) denoting the complementary error function and $P_{\text {leak }}$ expressed in Watts.

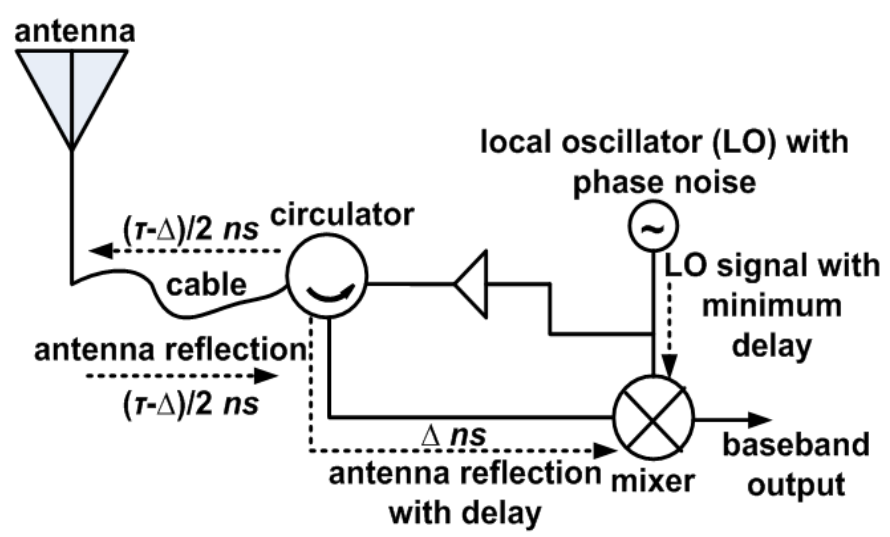

Fig. 4. Delayed antenna reflection depicting phase noise conversion to equivalent amplitude noise. All delay measurements are in nanoseconds (ns).

Often, an operating BER threshold value is $B E R_{t h}=10^{-3}$ at $T=300 \mathrm{~K}$ [12]. If $\Delta V=\left|V_{1}-V_{2}\right|$, then Fig. 3 depicts the necessary $\Delta V=11 \mu \mathrm{V}$ to achieve the desired BER of $10^{-3}$ for $B W=640 \mathrm{kHz}$. For $B W=40 \mathrm{kHz}, \Delta V=2.75 \mu \mathrm{V}$. Note that $640-40 \mathrm{kHz}$ denotes the range of the uplink signal, corresponding to binary modulation at rates of $640(\max )-40$ (min) Kbps as specified in the EPC Global standard [13].

The uplink range estimation is undertaken based on the necessary $\Delta V$ for a specific tag bandwidth. By employing equations (21), (23), (24) and (25), it can be derived that

$$
\Delta V=\frac{Z_{0}\left|V_{O C}\right|\left|\alpha_{r d}\right|\left|\alpha_{t a g} \| m_{1}-m_{2}\right|}{2 R_{\text {ant }} \lambda r_{\text {read }}\left(1+m_{1}\right)\left(1+m_{2}\right)}
$$

In equation (28), $V_{O C}$ should be replaced with

$$
V_{\text {OC }}=\frac{\alpha_{\text {tag }}}{2 r_{\text {read }}}\left(\frac{Z_{0} \eta P_{\text {eirp }}}{\pi}\right)^{1 / 2}
$$

yielding 


$$
\Delta V=\frac{Z_{0}\left|\alpha_{r d}\right|\left|\alpha_{\text {tag }}\right|^{2}\left|m_{1}-m_{2}\right|}{4 \lambda R_{\text {ant }} r_{\text {read }}^{2}\left(1+m_{1}\right)\left(1+m_{2}\right)}\left(\frac{Z_{0} \eta P_{\text {eirp }}}{\pi}\right)^{1 / 2}
$$

Thus, based on the BER requirement, the read (uplink) range $D_{\text {read }}$ is

$$
\begin{array}{r}
D_{\text {read }}=\frac{\left|\alpha_{\text {tag }}\right|}{2} \sqrt{\frac{Z_{0}\left|\alpha_{r d}\right|\left|m_{1}-m_{2}\right|}{\lambda \Delta V R_{\text {ant }}\left(1+m_{1}\right)\left(1+m_{2}\right)}} \\
\left(\frac{Z_{0} \eta P_{\text {eirp }}}{\pi}\right)^{1 / 4}
\end{array}
$$

\section{RANGE MAXIMIZATION}

A link budget analysis is undertaken to characterize the read range $D_{\text {read }}$ and write range $D_{\text {write }}$ for an RFID system operating at $915 \mathrm{MHz}$ with $640 \mathrm{kHz}$ tag bandwidth. Specifically, an analytical expression for the optimal impedance modulation indices $m_{1}$ and $m_{2}$ that concurrently maximize both $D_{\text {read }}$ and $D_{\text {write }}$ is derived. The maximum reader-tag distance is $D_{\text {range }}=\min .\left\{D_{\text {read }}, D_{\text {write }}\right\}$. Thus, range maximization of RFID tags is commensurate with (a) first equalizing $D_{\text {read }}$ and $D_{\text {write }}$, and (b) then maximizing this common range.

\section{A. Equalization of Read and Write Ranges}

Three different situations arise based on the chosen modulation scheme - (a) parallel only, (b) series only, and (c) alternate parallel and series (mixed). The corresponding write ranges are defined as $D_{\text {write }}^{\text {parallel }}, D_{\text {write }}^{\text {series }}$ and $D_{\text {write }}^{\text {mixed }}$ in equations (18), (19) and (20) respectively.

1) Parallel Modulation: Equating $D_{\text {write }}^{\text {parallel }}$ from equation (18) and $D_{\text {read }}$ from equation (31), the following equation may be used to select $m_{2}$ for a chosen value of $m_{1}$ in the range $0<m_{1} \leq 1$ such that $m_{2}<m_{1}$

$$
m_{2}=\frac{-B \pm \sqrt{B^{2}-4 A C}}{2 A}
$$

where

$$
\begin{aligned}
& A=F+2 m_{1} F+2 m_{1}^{2} F+1+m_{1} \\
& B=2 m_{1}^{2} F-m_{1}^{2}+1 \\
& C=m_{1}^{2} F-m_{1}-m_{1}^{2}
\end{aligned}
$$

and

$$
F=\frac{\lambda \Delta V}{4 P_{\text {tag }}^{0}\left|\alpha_{r d}\right|}\left(\frac{\eta P_{\text {eirp }}}{\pi Z_{0}}\right)^{1 / 2}
$$

Though equation (32) may yield two possible values of $m_{2}$, the correct value is chosen such that $m_{2}>0$ and $m_{2}<m_{1}$. It is also possible to select $m_{1}<m_{2}$, and owing to symmetry in the relationship between $m_{1}$ and $m_{2}$, their values just need to be interchanged.
2) Series Modulation: Equating $D_{\text {write }}^{\text {series }}$ from equation (19) and $D_{\text {read }}$ from equation (31), the following equation may be used to select $m_{1}$ for a chosen value of $m_{2}$ in the range $m_{2} \geq 1$ such that $m_{1}>m_{2}$

$$
m_{1}=\frac{-B \pm \sqrt{B^{2}-4 A C}}{2 A}
$$

where

$$
\begin{aligned}
& A=F-1-m_{2} \\
& B=2 F+m_{2}^{2}-1 \\
& C=2 F+m_{2}^{2} F+2 m_{2} F+m_{2}+m_{2}^{2}
\end{aligned}
$$

and $F$ is defined in equation (34).

Again, equation (35) may yield two possible values of $m_{1}$, and the correct value is chosen such that $m_{1}>1$ and $m_{1}>$ $m_{2}$. It is also possible to select $m_{1}<m_{2}$, and symmetry in the relationship between $m_{1}$ and $m_{2}$ may be directly exploited to interchange values.

3) Alternate Parallel and Series Modulation: Equating $D_{\text {write }}^{\text {mixed }}$ from equation (20) and $D_{\text {read }}$ from equation (31), the following equation may be used to select $m_{1}$ for a chosen value of $m_{2}$ in the range $m_{2} \geq 1$ such that $m_{1}<m_{2}$ and $0<m_{1}<1$

$$
m_{1}=\frac{-B \pm \sqrt{B^{2}-4 A C}}{2 A}
$$

where

$$
\begin{aligned}
& A=2 F+2 m_{2} F+m_{2}^{2} F+1+m_{2} \\
& B=2 F-m_{2}^{2}+1 \\
& C=F-m_{2}-m_{2}^{2}
\end{aligned}
$$

and $F$ is defined in equation (34).

Even though equation (36) may yield two possible values of $m_{1}$, and the correct value is chosen such that $0<m_{1}<$ 1 and $m_{1}<m_{2}$. It is also possible to select $m_{1}>m_{2}$, and symmetry in the relationship between $m_{1}$ and $m_{2}$ may be directly exploited to interchange values. This interchange implies series modulation in statel and parallel modulation in state 2 .

\section{B. Range Maximization}

The range maximization problem is essentially a constrained optimization problem that aims to maximize read (or write) range and simultaneously equate read and write ranges. Sequential quadratic programming within the Matlab environment is used for optimization. Let $D_{\text {write }}$ be a generic reference to $D_{\text {write }}^{\text {paralle }}, D_{\text {write }}^{\text {series }}$ or $D_{\text {write }}^{\text {mixed }}$. It must be noted that both $D_{\text {read }}$ and $D_{\text {write }}$ are functions of $m_{1}$ and $m_{2}$, and are explicitly referred to as $f^{(\text {read })}\left(m_{1}, m_{2}\right)$ and $f^{\text {(write) }}\left(m_{1}, m_{2}\right)$ for a complete mathematical description of the problem as

$$
\begin{gathered}
\text { Maximize } f^{(\text {read })}\left(m_{1}, m_{2}\right) \\
\text { subject to } \\
f^{(\text {read })}\left(m_{1}, m_{2}\right)-f^{(\text {write })}\left(m_{1}, m_{2}\right)=0
\end{gathered}
$$


Since $f^{(\text {read })}\left(m_{1}, m_{2}\right)$ and $f^{(\text {write })}\left(m_{1}, m_{2}\right)$ are estimated during the optimization procedure with only $m_{1}$ and $m_{2}$ as variables of interest, it is necessary to discuss the fixed values assumed by $Z_{0}, P_{\text {eirp }}, \eta, \lambda, \Delta V, R_{a n t},\left|\alpha_{t a g}\right|,\left|\alpha_{r d}\right|$ and $P_{t a g}^{0}$ in equations (18), (19), (20) and (31). The first four terms are $Z_{0}=377 \Omega, P_{\text {eirp }}=4 \mathrm{~W}, \eta=0.5$ (reader antenna assumed to have $0 \mathrm{~dB}$ axial ratio) and $\lambda=0.32$ meters at $915 \mathrm{MHz}$ operation frequency. For $B W=640 \mathrm{kHz}, \Delta V=11 \mu \mathrm{V}$.

The magnitude of the vector effective length of the tag antenna for a given system geometry (i.e. as a function of reader position $(r, \theta, \phi))$, and antenna resistance are best estimated by use of electromagnetic (EM) simulation. In this work, the 3D EM full-wave field solver that measures these terms is PhysWAVE ${ }^{\complement}$ [14]. Thus, $\left|\alpha_{\text {tag }}\right|$ is measured as 0.1 for a half-wavelength dipole employed as the tag antenna, with the reader positioned in its broadside direction. For the same tag antenna at $915 \mathrm{MHz}, R_{\text {ant }} \approx 76 \Omega$. Once $\left|\alpha_{\text {tag }}\right|$ is measured, equation (26) is employed to calculate $\left|\alpha_{r d}\right|$ for the reader antenna. Since the reader has been positioned in the broadside direction of the tag with $G_{t a g}=2 \mathrm{dBi},\left|\alpha_{r d}\right|=0.12$ with an effective receive antenna gain of $3 \mathrm{dBi}$ after accounting for polarization mismatch on the uplink. The optimal solution is determined for a chosen tag sensitivity, $P_{t a g}^{0}$.

The maximization of read range is subject to the nonlinear equality constraint equating read and write ranges. Thus, if the optimal values are $m_{1}^{\star}$ and $m_{2}^{\star}$, then the maximum operable reader-tag distance $D_{\text {range }}$ is

$$
\begin{array}{r}
D_{\text {range }}=\frac{\left|\alpha_{\text {tag }}\right|}{2} \sqrt{\frac{Z_{0}\left|\alpha_{r d}\right|\left|m_{1}^{\star}-m_{2}^{\star}\right|}{\lambda \Delta V R_{\text {ant }}\left(1+m_{1}^{\star}\right)\left(1+m_{2}^{\star}\right)}} \\
\left(\frac{Z_{0} \eta P_{\text {eirp }}}{\pi}\right)^{1 / 4}
\end{array}
$$

The ranges of values of $m_{1}$ and $m_{2}$ within which $m_{1}^{\star}$ and $m_{2}^{\star}$ will lie depend on the chosen modulation scheme and are enumerated as

- Parallel modulation: $0 \leq m_{1} \leq 1$ and $0 \leq m_{2}<1$

- Series modulation: $m_{1} \geq 1$ and $m_{2}>1$

- Mixed modulation: $0 \leq m_{1} \leq 1$ and $m_{2}>1$

The question now arises - are these ranges of $m_{1}$ and $m_{2}$ attainable for any choice of tag sensitivity? Based on equations (32)-(38), it becomes obvious that the factor $F$ in equation (34) is inversely proportional to tag sensitivity $P_{t a g}^{0}$, while all other contributing factors including $\Delta V\left(B E R_{t h}=10^{-3}\right)$ remain constant. Since $F$ has a direct impact on the discriminant of the quadratic equations (32), (35) and (37), the appropriate ranges of $m_{1}$ and $m_{2}$ will depend exclusively on tag sensitivity.

The approximate ranges of $m_{1}$ and $m_{2}$ for parallel modulation are depicted in Table I along with the corresponding tag sensitivity measure. The ranges are interchangeable based on symmetry.

For series modulation, the ranges of $m_{1}$ and $m_{2}$ are both $[1, \infty]$, and remain unaffected by tag sensitivity.

However, as depicted in Table II, these ranges undergo drastic changes for mixed parallel and series modulation as tag sensitivity improves. Interchangeability of ranges remains a viable option.
TABLE I

POSSIBLE RANGES OF $m_{1}$ AND $m_{2}$ FOR PARALLEL MODULATION

\begin{tabular}{|c|c|c|}
\hline Tag sensitivity & Range of $m_{1}$ & Range of $m_{2}$ \\
\hline \hline $0 \mathrm{dBm}$ & {$[0,1]$} & {$[0,0.999]$} \\
\hline$-10 \mathrm{dBm}$ & {$[0,1]$} & {$[0,0.994]$} \\
\hline$-25 \mathrm{dBm}$ & {$[0,1]$} & {$[0,0.829]$} \\
\hline$-35 \mathrm{dBm}$ & {$[0,1]$} & {$[0,0.254]$} \\
\hline
\end{tabular}

\section{Impact of Technology Scaling}

The improvement in reader-tag operable distance, $D_{\text {range }}$, can be attributed to technology scaling that improves tag sensitivity [5]. A comprehensive overview of tag sensitivity is provided in [2]. Table III outlines the dependence of optimal impedance modulation indices for either parallel or series modulation on tag sensitivity. No modulation is necessary when either $m_{1}^{\star}=1$ or $m_{2}^{\star}=1$, and the entry corresponding to the modulating resistance in this state is omitted from the table. The important observations from Table III are:

TABLE II

POSSIBLE RANGES OF $m_{1}$ AND $m_{2}$ FOR MIXED MODULATION

\begin{tabular}{|c|c|c|}
\hline Tag sensitivity & Range of $m_{1}$ & Range of $m_{2}$ \\
\hline \hline $0 \mathrm{dBm}$ & 0.999 & 1 \\
\hline$-10 \mathrm{dBm}$ & {$[0.993,0.999]$} & {$[1,1.007]$} \\
\hline$-25 \mathrm{dBm}$ & {$[0.813,0.993]$} & {$[1,1.23]$} \\
\hline$-35 \mathrm{dBm}$ & {$[0.243,0.999]$} & {$[1,4.12]$} \\
\hline
\end{tabular}

- Irrespective of the choice of modulation, one optimal index is always $m_{1}^{\star}=1$ (or, alternatively, $m_{2}^{\star}=1$ from symmetry). The explanation lies in the fact that the tag write range is maximized for conjugate match. Hence, equalization of read and write ranges for $m_{1}^{\star}=1$ is the obvious choice, with $m_{2}^{\star}$ based on uplink and downlink range trade-off.

- If $m_{2}^{*}$ is denoted by $m_{2}^{\text {parallel }}$ for parallel modulation and $m_{2}^{\text {series }}$ for series modulation, then their relationship may be defined as $m_{2}^{\text {series }}=1 / m_{2}^{\text {parallel }}$. It must be mentioned that the choice of $m_{2}^{\star}$ for parallel and series modulation equalize power supplied to the tag IC as well as backscattered power to the reader between them only when $m_{1}^{*}=1$. If $m_{2}$ is replaced by $1 / m_{2}$ (with $m_{1}=1$ ) in equations (20) and (31) for $D_{\text {write }}^{\text {series }}$ and $D_{\text {read }}$ respectively, then the former transforms into $D_{\text {write }}^{\text {parallel }}$ while $D_{\text {read }}$ remains unchanged. Thus, range maximization may be achieved using either parallel or series modulation. Design consideration such as ease of realization of on-chip modulating resistance $R_{m_{2}^{\star}}^{\text {mod }}$ may eventually dictate the choice of the modulation scheme.

- Mixed modulation simply reduces to parallel modulation for range maximization. An equal mismatch condition for ASK does not maximize range.

- The choices of (a) $m_{1}=1$ and $m_{2}=0$ for parallel modulation $[3,15]$, or (b) equal mismatch such that $m_{1}=$ $1 / m_{2}\left(\rho_{1}=-\rho_{2}\right)$ for mixed modulation are always suboptimal for range maximization. This important issue has been consistently overlooked in the literature on RFID system deployment. As a baseline comparison, Table IV depicts the achievable $D_{\text {range }}$ for choice (a). A $D_{\text {range }}$ 
TABLE III

IMPACT OF TECHNOLOGY SCALING

\begin{tabular}{|c|c|c|c|c|c|c|c|c|c|c|}
\hline \multirow[b]{2}{*}{$P_{t a g}^{0}$} & \multicolumn{3}{|c|}{ Parallel Modulation } & \multicolumn{3}{|c|}{ Series Modulation } & \multicolumn{3}{|c|}{ Mixed Modulation } & \multirow[b]{2}{*}{$D_{\text {range }}$} \\
\hline & $m_{1}^{\star}$ & $m_{2}^{\star}$ & $\begin{array}{c}R_{m_{2}^{\star}}^{\bmod } \\
m^{\star}\end{array}$ & $m_{1}^{\star}$ & $m_{2}^{\star}$ & $\begin{array}{c}R_{m_{2}^{\star}}^{\bmod } \\
m^{\star}\end{array}$ & $m_{1}^{\star}$ & $m_{2}^{\star}$ & $\begin{array}{c}R_{m_{1}^{\star}}^{\bmod } \\
\end{array}$ & \\
\hline$-10 \mathrm{dBm}$ & $\overline{1}$ & 0.994 & $11.7 \mathrm{k} \Omega$ & $\overline{1}$ & 1.007 & $0.5 \Omega$ & $\overline{0.994}$ & 1 & $11.7 \mathrm{k} \Omega$ & 3.14 meters \\
\hline$-25 \mathrm{dBm}$ & 1 & 0.829 & $370 \Omega$ & 1 & 1.206 & $15.6 \Omega$ & 0.829 & 1 & $370 \Omega$ & 16.86 meters \\
\hline$-35 \mathrm{dBm}$ & 1 & 0.254 & $25.84 \Omega$ & 1 & 3.936 & $223.5 \Omega$ & 0.254 & 1 & $25.84 \Omega$ & 42.6 meters \\
\hline
\end{tabular}

of 12.49 meters for $P_{t a g}^{0}=-25 \mathrm{dBm}$ in Table IV closely matches the 12 meters achieved in [5] for $4 \mathrm{~W}$ EIRP.

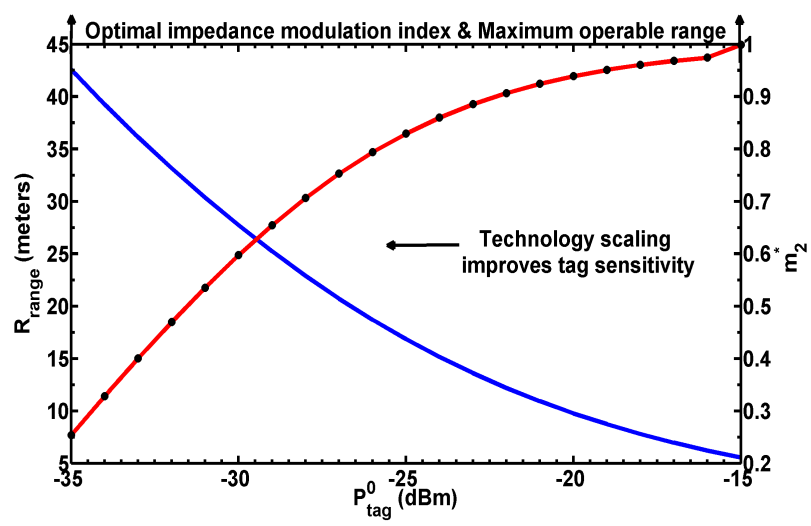

Fig. 5. Optimal impedance modulation index $m_{2}^{\star}\left(=m_{2}^{\text {parallel }}\right)$ and maximum operable range $D_{\text {range }}$ each as a function of tag sensitivity.

Figure 5 outlines both $D_{\text {range }}$ and $m_{2}^{\star}\left(=m_{2}^{\text {parallel }}\right)$ as a function of tag sensitivity. Thus, range improvement is empowered by technology scaling.

With further improvement in tag sensitivity $\left(P_{\text {tag }}^{0}<-35\right.$ $\mathrm{dBm}$ ), the tag becomes uplink limited. Semi-passive tags have sensitivities around $-40 \mathrm{dBm}$ [16]. Thus, for semi-passive tags, the maximum range is simply the read range, $D_{\text {read }}$, for $m_{1}=1$ and $m_{2}=0$. This choice of $m_{1}$ and $m_{2}$ maximizes backscattered power, and involves a conjugate match in statel in conjunction with shorted IC resistance in state2.

TABLE IV

MAXIMUM RANGE FOR PARALLEL MODULATION

\begin{tabular}{|c|c|c|c|}
\hline Tag sensitivity & $m_{1}$ & $m_{2}$ & $D_{\text {range }}$ \\
\hline \hline$-10 \mathrm{dBm}$ & 1 & 0 & 2.22 meters \\
\hline$-25 \mathrm{dBm}$ & 1 & 0 & 12.49 meters \\
\hline$-35 \mathrm{dBm}$ & 1 & 0 & 39.5 meters \\
\hline
\end{tabular}

\section{REFLECTIONS AND CONCLUSIONS}

Figure 5 emphasizes the fact that for fixed reader sensitivity, an enhancement in tag sensitivity improves $D_{\text {range }}$ for RFID tags. Are any other degrees of freedom available to designers to further improve $D_{\text {range}}$ ?

The key to improving $D_{\text {range }}$ lies in careful design of the tag antenna and IC. Typically, the goal of tag antenna design is to conjugate match it to the IC impedance [1]. A marginally better tag design is proposed when $P_{t a g}^{0}=-35$ $\mathrm{dBm}$ and parallel modulation is employed for backscatter. It is assumed that the antenna resistance and nominal IC resistance are intentionally mismatched such that $R_{a n t} \neq R_{I C}$.

The tag IC resides in two impedance states state 1 and state 2 where the impedances are, respectively,

$$
\begin{aligned}
& Z_{I C}^{\text {state } 1}=m_{1} R_{a n t}-j \omega L_{a n t} \\
& Z_{I C}^{\text {state } 2}=m_{2} R_{a n t}-j \omega L_{a n t}
\end{aligned}
$$

with $m_{2}<m_{1}$ for parallel modulation. In this case, however, $Z_{I C}^{\text {state } 1}$ is the nominal IC impedance and $Z_{I C}^{\text {state } 2}$ is the modulated impedance. Thus, the parallel modulating resistance that transforms $Z_{I C}^{\text {state } 1}$ to $Z_{I C}^{\text {state2 }}$ is

$$
R_{\text {parallel }}^{\text {mod }}=\left(\frac{m_{1} m_{2}}{m_{1}-m_{2}}\right) R_{a n t}
$$

The actual usable power delivered to the tag IC in state1 and state 2 are, respectively,

$$
\begin{gathered}
P_{\text {usable }, I C}^{\text {state } 1}=\frac{\left|V_{O C}\right|^{2}}{2 R_{a n t}} \frac{m_{1}}{\left(1+m_{1}\right)^{2}} \\
P_{\text {usable }, I C}^{\text {state } 2}=\frac{\left|V_{O C}\right|^{2}}{2 R_{a n t}} \frac{m_{2}^{2}}{m_{1}\left(1+m_{2}\right)^{2}}
\end{gathered}
$$

Thus, the write range is

$$
\begin{aligned}
D_{\text {write }}^{\text {parallel }}= & \left.=\frac{\left|\alpha_{\text {tag }}\right|}{4}\right]\left(\frac{Z_{0} \eta P_{\text {eirp }}}{\pi R_{\text {ant }} P_{\text {tag }}^{0}}\right)^{1 / 2} \\
& \sqrt{\left[\frac{m_{1}}{\left(1+m_{1}\right)^{2}}+\frac{1}{m_{1}}\left(\frac{m_{2}}{1+m_{2}}\right)^{2}\right]}
\end{aligned}
$$

The read range estimation is still based on equation (31). The optimal indices turn out to be $m_{1}^{\star}=1.41$ and $m_{2}^{\star}=0.39$, and the maximum achievable range is 43.16 meters. The optimal solution, $m_{1}^{*}$, implies that the tag should be designed for $R_{I C} \approx 107 \Omega$. However, $D_{\text {range }}$ improves by only 0.6 meters for $P_{t a g}^{0}=-35 \mathrm{dBm}$. Introducing an intentional mismatch between $R_{a n t}$ and $R_{I C}$ yields range improvement, but the overall gain in RFID performance should justify tag re-design. In the aforementioned case, tag re-design is not necessary since it offers negligible range improvement. It must be noted that $m_{1}^{\star} \neq 1 / m_{2}^{\star}\left(\rho_{1} \neq-\rho_{2}\right)$, and this implies that an equal mismatch condition $[3,4]$ is sub-optimal for range maximization.

The magnitude of the vector effective lengths $\alpha_{t a g}$ and $\alpha_{r d}$ associated with tag and reader antennas improve with an increase in their respective antenna gains, $G_{t a g}$ and $G_{r x}$. This improves both write and read ranges. A different antenna type 
such as a patch antenna may yield a higher gain [1] than the typical dipole considered in this paper. Thus, designers have flexibility in improving $D_{\text {range }}$ based on aforementioned design choices.

This work demonstrates the need to consider the impact of impedance modulation indices on the read/write range for passive RFID tags. Using a link budget analysis leveraged by EM simulation, this paper investigates the choice of ASK impedance modulation indices that maximize the operating range as a function of key system parameters - notably the tag sensitivity and bit error rate at the reader. Technology scaling will continue to improve tag sensitivity, implying that existing values of impedance modulation indices must be modified. The analysis put forth in this paper suggests how on-chip parallel or series modulating resistances may be used to achieve optimum reader-tag distances.

\section{REFERENCES}

[1] D. Dobkin, The RF in RFID. Elsevier Inc., 2008.

[2] P. Pursula, "Analysis and Design of UHF and Millimetre Wave Radio Frequency Identification," VTT Publications 701, 2009.

[3] U. Karthaus and M. Fischer, "Fully Integrated Passive UHF RFID Transponder IC With 16.7- $\mu \mathrm{W}$ Minimum RF Input Power," IEEE Journal of Solid-State Circuits, vol. 38, pp. 1602-1608, October 2003.

[4] G. D. Vita and G. Iannacone, "Design Criteria for the RF Section of UHF and Microwave Passive RFID Transponders," IEEE Transactions on Microwave Theory and Techniques, vol. 53, pp. 2978-2990, September 2005.

[5] J. Curty, N. Joehl, C. Dehollain, and M. J. Declercq, "Remotely Powered Addressable UHF RFID Integrated System," IEEE Journal of Solid-State Circuits, vol. 40, pp. 2193-2202, November 2005.

[6] N. Cho, S. J. S. S. Kim, S. Kim, and H. J. Yoo, "A 5.1- $\mu$ W UHF RFID Tag Chip Integrated with Sensors for Wireless Environmental Monitoring," Proceedings of ESSCIRC, pp. 279-282, September 2005.

[7] Y. Xi, H. Kim, H. Cho, M. Kim, S. Jung, C. Park, J. Kim, and Y. Yang, "Optimal ASK Modulation Scheme for Passive RFID Tags Under Antenna Mismatch Conditions," IEEE Transactions on Microwave Theory and Techniques, vol. 57, pp. 2337-2343, October 2009.

[8] F. Fuschini, C. Piersanti, F. Paolazzi, and G. Falciasecca, "Analytical Approach to the Backscattering from UHF RFID Transponders," IEEE Antennas and Wireless Propagation Letters, vol. 7, pp. 33-35, 2008.

[9] J. D. Kraus, Antennas. McGraw-Hill Book Company Inc., 1950.

[10] R. C. Hansen, "Relationships Between Antennas as Scatterers and as Radiators," Proceedings of the IEEE, vol. 77, pp. 659-662, May 1989.

[11] C. Mutti and A. Wittneben, "Robust Signal Detection in Passive RFID Systems," Proceedings of the First International EURASIP Workshop RFID Technology, Austria, pp. 39-42, September 2007.

[12] F. Fuschini, C. Piersanti, F. Paolazzi, and G. Falciasecca, "On the Efficiency of Load Modulation in RFID Systems Operating in Real Environment," IEEE Antennas and Wireless Propagation Letters, vol. 7 pp. 243-246, 2008.

[13] " $E P C^{T M}$ Radio-Frequency Identity Protocols Class-1 Generation-2 UHF RFID Conformance Requirements Version 1.0.4," EPCglobal Inc., July 2006.

[14] "PhysWAVE 3.8 User's Guide," Physware Inc., April 2009. http://www.physware.com/.

[15] A. P. Sample, D. J. Yeager, P. S. Powledge, A. V. Mamishev, and J. R. Smith, "Design of an RFID-Based Battery-Free Programmable Sensing Platform," IEEE Transactions on Instrumentation and Measurement, vol. 57, pp. 2608-2615, November 2008.

[16] "Passive, Battery-assisted Passive and Active Tags: A Technical Comparison," Intelleflex Corporation, 2005. http://www.intelleflex.com/. 